\title{
SELECTED ISSUES CONCERNING DEGRADATION OF MATERIAL IN THE PRODUCTION OF INJECTION MOLDED PLASTIC COMPONENTS
}

\author{
Marek JAŁBRZYKOWSKI* \\ *Faculty of Mechanical Engineering, Department of Materials Science and Biomedical Engineering, Bialystok University of Technology, \\ ul. Wiejska 45C, 15-351 Białystok, Poland \\ m.jalbrzykowski@pb.edu.pl
}

received 14 July 2015, revised 14 July 2016, accepted 18 July 2016

\begin{abstract}
This paper presents the problem of thermal degradation of thermoplastic materials processed using the injection method. Attention was paid to the issue of the optimal selection of a dye for modifying the base materials. For the selected materials and dyes, derivatograph tests were performed in order to assess their thermal characteristics and breakdown kinetics. Additionally, tribological tests and microscope observations of selected samples were performed. The obtained test results suggest a diverse level of thermal processes in the analyzed materials. This is crucial for the appropriate selection of dyes for plastic materials. As it turned out, the tribological properties of materials can also influence the technological quality of the injected alloy.
\end{abstract}

Key words: Dyes For Plastic Materials, Thermal Degradation, Thermogravimetric Tests, Tribological Tests

\section{INTRODUCTION}

The problem of the functional quality of injection molded components is an everyday issue in all injection molding plants (Zawistowski and Zięba, 1999). The functional quality of the produced components shapes, among others, the level of effective productive work, the number of parts sold, or the amount of production scrap, both internal and external (Zawistowski and Zięba, 1999; Chun, 1999). It is a well-known fact that the functional quality mentioned above can depend on numerous factors, among which there are, for example, the processed material (Jałbrzykowski, 2012), the construction of the injection mold (Matin et al., 2012; Jałnrzykowski and Nachimowicz, 2009), or the parameters of the injection process (Zawistowski and Zięba, 1999; Chun, 1999; Chen et al., 2016; Wu and Chen, 2006) (Fig. 1). As far as the processed material is concerned, the following aspects can be mentioned, e.g. the problem of the quality of pellets, even those from the same batch; the problem of appropriate drying of material, or the problem of appropriate selection of dye and the homogeneity of components in the plasticizing system (Zawistowski and Zięba, 1999; Chun, 1999; Jałbrzykowski, 2012; Matin et al., 2012).

The data obtained from Bialystok's entrepreneurs, in addition to own experience, allow to conclude that problems concerning the quality of components in a stable process are a common occurrence, even if no quality problems have been noticed previously. To illustrate, the process has up to now been repeatable, the machine operation has been stable, and the tool underwent control twice during a production shift. The material was fed from the same batch, prepared for the process in the same way, i.e. the same temperature and drying time, on the same dryers; the same proportional content of dye, determined using agitators of the same type. Yet, despite all these precautions, quality problems of components suddenly appeared. It is probable that this situation is caused by the material, whose physical and chemical properties may not be completely uniform (even in a small volume of material). These differences, although slight, can influence the quality of the produced components. It is worth adding that in most cases these problems tended to disappear spontaneously after several minutes.

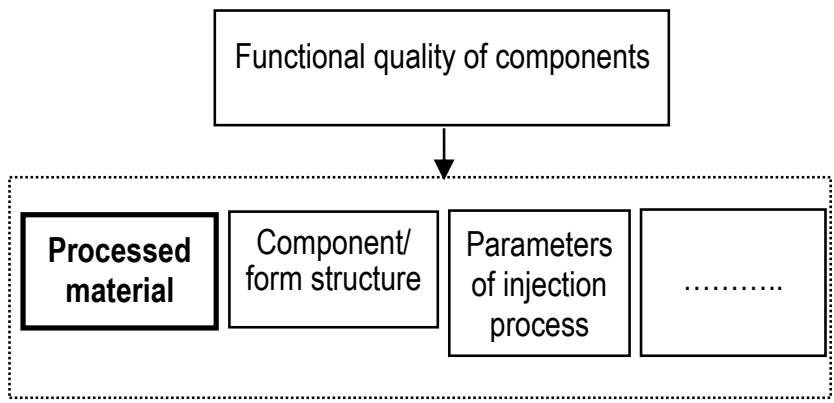

Fig. 1. Example factors influencing the functional quality of injection molded components

The issue of appropriate drying of material is obvious and no comments are in fact necessary. It can only be mentioned that the most common defect resulting from insufficient drying of material are the so-called 'silver streaks', as production nomenclature has it.

The author's interest was aroused particularly by the issue of dye selection (in the case of mixing the base material with a dye) and the homogenization of plastic. Basically, the issues concerning dye selection are connected with three aspects, i.e. the selection of a polymer matrix on the basis of which the dye is produced, the method for measuring the appropriate quantity of dye (gravimetric, volumetric, or manual, if necessary), and the homogenization of alloy in the plasticizing system. As industry practice shows, it is not enough to purchase a dye to modify 
a specific material. This problem should be analyzed more thoroughly. It often happens that a dye purchased to modify material A was produced on the basis of material B. In such situations, manufacturers of thermoplastic components do not usually make mistakes concerning the compatibility of mixtures. However, the aspect of different ranges of processing temperatures, including the thermal degradation of the prepared ingredients, is often neglected. In extreme cases, this state may lead either to a lack of homogeneity of the plastic alloy (uneven coloring, smudges, nonplasticized pellets, etc.), or to local degradation of the mixture ingredients, despite the fact that a specific dye, dedicated specially to the modification of a specific material was used. As an example, Fig. 2 shows several samples with visible defects resulting from non-homogenous plasticizing of an alloy.

a)

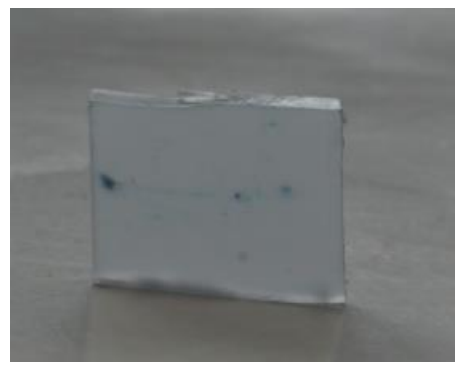

b)

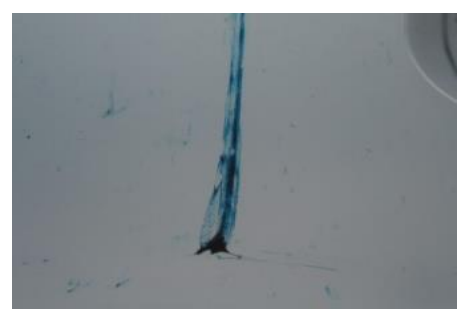

c)

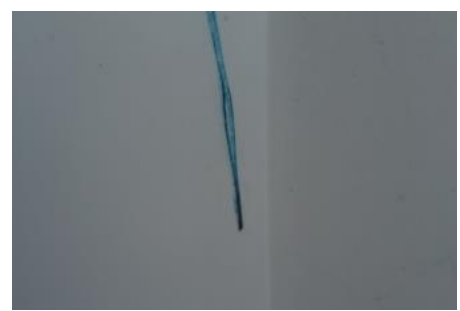

Fig. 2. Examples of defects of components (macrographic): a) section of a component $b$ ) and $c$ ) clearly visible dye smudges in the wall of the component

The issues mentioned above prompted the author to undertake a deeper analysis of situations typically encountered in the industry in the course of the production process. The aim of this paper was to assess the technological quality of selected samples made from plastic (PM- plastic materials) in the area of their thermal and tribological properties (Brachet et al., 2008; Torres et al., 2000; Badia et al., 2009; Navarro et al., 2003; Al-Salem et al., 2010).

It should be emphasized once again that the subject matter described above is still present to a smaller or greater extent in every injection moulding plant and thus is currently very much an issue of relevance (www2.dupont.com, http://www.plastech.pl, http://www.wadim.com.pl, https://www.elsevier.com, Botos et al., 2014; Bucella et al., 2015; Laurence and McKeen, 2007; Kayvon, 2015). The types of machines and raw material used, or the method of process realization is of no importance here. It can also be added that the influence of dye becomes visible not only during visual assessment, but is also reflected in other characteristics of injected parts. A common problem is, for example, cracking of mouldings despite the optimized technological process. This problem is a deeper issue that requires, above all, an in-depth analysis of mechanisms of the described phenomena. As traditional technological actions do not yield sufficient effects, a scientific explanation and solution to the described problems needs to be sought. These issues will be dealt with and analyzed if further papers. In this light, the issue becomes extremely significant. As one of the most important ones, it is the subject of studies by, among many others: DuPont (www2.dupont.com), Plastech (http://www.plastech.pl), Wadim Plast (http://www.wadim.com.pl). This subject matter has also been indicated on websites containing scientific studies (https://www.elsevier.com); however, explanation of the causes of the described phenomena is highly insufficient.

\section{MATERIALS AND METHODOLOGY OF RESEARCH}

\subsection{Materials used for the tests}

For the tests, PM samples were received in the form of pellets, directly from the production line of one of Bialystok's companies. These were four samples of materials: the base material (polycarbonate) and three kinds of dye. Respecting the company's secrets, the data concerning the materials was coded in the following way: base material - PC, dyes: blue - B, green - $G$, orange - $\mathrm{O}$ (Fig. 3). The breakdown temperature of the basic material and the dyes was adopted as the main indicator of the technological quality of the received PM's. Additionally, tests of the tribological properties of the received PM samples were performed.

The impetus to perform the tests presented in this paper were the constant problems to obtain an appropriate visual quality of the components made from PC dyed with the additives mentioned earlier.

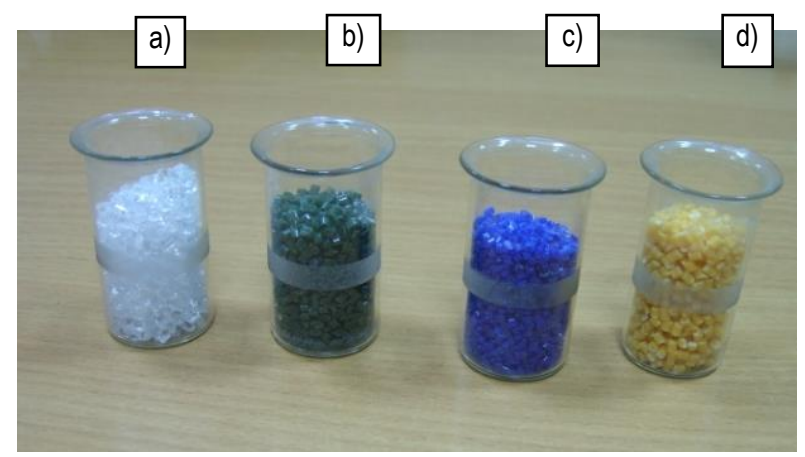

Fig. 3. Samples of materials received for the tests: a) PC, b) dye G, c) dye B, d) dye 0

\subsection{Methodology of tests}

The aim of this paper was to assess the breakdown temperatures of the received PM's, focusing especially on the possibility of their earlier degradation. 'Earlier degradation' is understood here as breakdown of PM during the normal process of its processing (at parameters stemming from the technological process). Owing to the current, 'severe' norms of quality control, a premature ther- 
mal breakdown of the processed materials disqualifies the produced components, e.g. in the area of visual quality, resulting in producing scrap. In order to solve this problem, thermogravimetric tests were performed as the basic tests. Supplementary to the basic tests, tribological tests whose purpose was to estimate the resistance to motion for the PM-steel system were performed. Increased values of resistance to motion can lead to the appearance of localized high temperature jumps, especially during the plasticizing process (in the slug-cylinder system), which can further intensify the process of PM breakdown. It should be mentioned that the problems connected with the visual quality of the produced components may arise due to the range of dye processing temperatures being to low in comparison with the base material.

The tests in the area of thermal properties were performed using a Paulik-Paulik-Erdey type derivatograph (Fig.4).

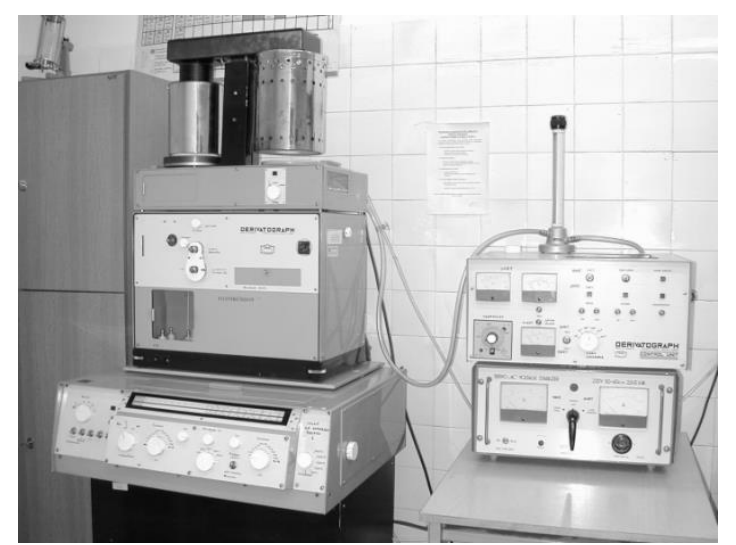

Fig. 4. View of the Paulik-Paulik-Erdey type apparatus (derivatograph) for thermogravimetric tests

Prior to the thermogravimetric tests, identical aliquots of materials weighing $\mathrm{m}=250 \mathrm{mg}$ were prepared. The derivatograph tests were performed at a constant velocity of heating $\mathrm{vt}_{\mathrm{t}}=5^{\circ} \mathrm{C} / \mathrm{min}$, until the maximum temperature of $\mathrm{T}=500$ ${ }^{\circ} \mathrm{C}$ was achieved. The parameters adopted for the tests were based, in the case of the velocity of heating, on literature data as well as standard recommendations. In the case of the maximum temperature of heating, real parameters of the injection process were used as guidelines, also taking into account the generally high temperature resistance of polycarbonate.

The subsequent analysis of the results of derivatograph tests prompted to perform additional tests, i.e. of thermal annealing in a constant temperature. These test were performed in temperatures of $T_{1}=280$ and $T_{2}=300{ }^{\circ} \mathrm{C}$. In the case of the first test, the material was annealed for $t=60 \mathrm{~min}$, while the second test was being carried out for $t=30 \mathrm{~min}$. The range of temperatures was adopted on the basis of the data concerning the recommended temperatures for the processing of the received PC, provided by the contractor. The time of annealing was meant to simulate the time period when the material stays in the cylinder of the injection molding machine during its down time. The aim of these tests was to check whether the time of annealing of the material in an increased temperature (even if the temperature did not exceed the breakdown temperature) has an influence on the degradation of material. The tests were performed using a furnace (Fig. 5). Prior to the tests, the furnace was annealed for $2 \mathrm{~h}$, in order to achieve a stable temperature. After the temperature had stabilized, samples of materials were placed in the furnace chamber (Fig. 6). a)

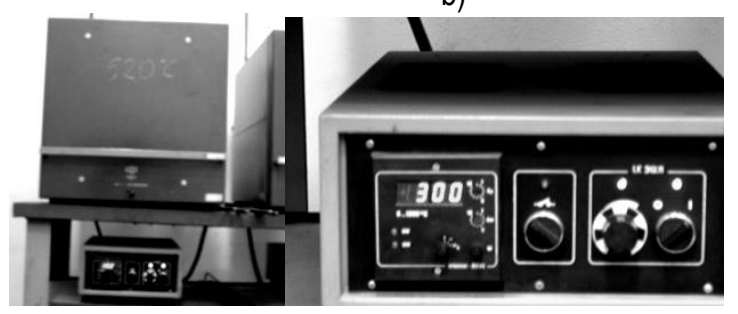

Fig. 5. The hardening furnace used for annealing tests: a) general view, b) view of the control system

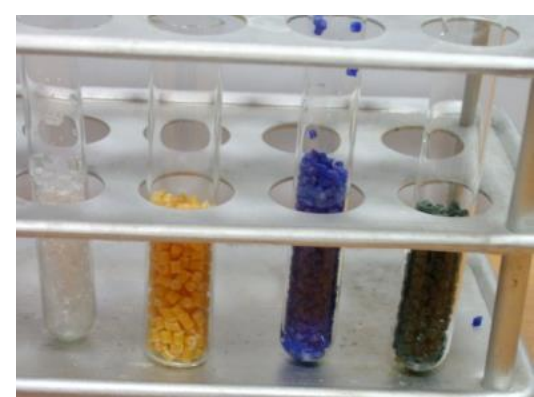

Fig. 6. An example of preparation of TS samples for the annealing tests

It should be added that before commencing the thermal tests and the tests of thermal annealing, the PM samples underwent conditioning in normal conditions for a time period of $24 \mathrm{~h}$.

After the thermogravimetric tests and the annealing tests, additional tests of the tribological properties of the received materials were performed. The tribological tests were performed using a pin-on-disc tester (Fig. 7). Samples prepared for the purpose of the tests were in the form of $\phi 1.5 \times 2 \mathrm{~mm}$ pins.

a)

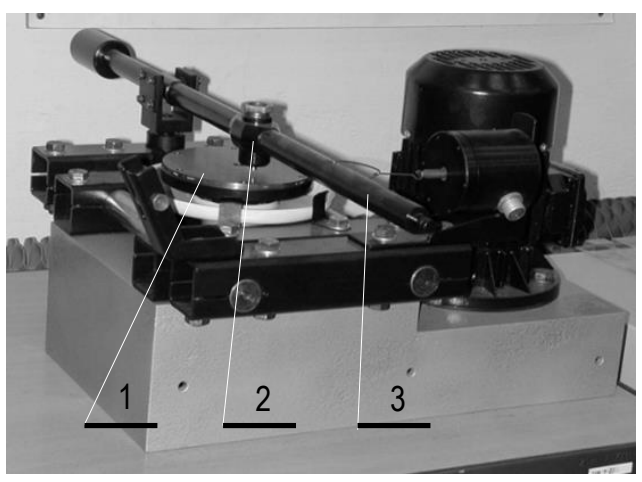

b)

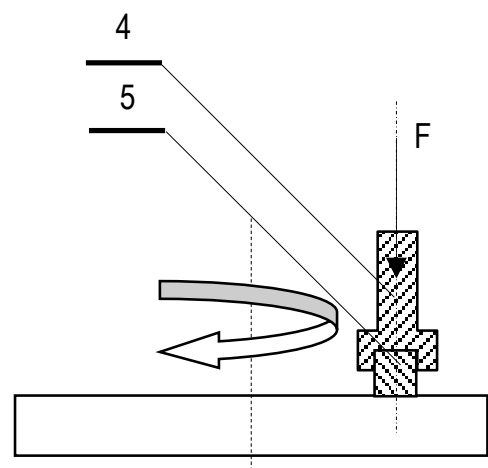

Fig. 7. Pin-on-disc tribometer: a) general view, b) scheme of the friction node; 1 - disc, 2 - pin handle, 3 - loading arm, 4 - pin fixing the sample, 5 - material sample 
Friction tests were performed at the following constant test parameters:

- load F= $160 \mathrm{~N}$,

- maximum friction time $t=3$ min or until sample destruction,

- sliding velocity $\mathrm{v}_{\mathrm{p}}=0,15 \mathrm{~m} / \mathrm{s}$.

\section{TEST RESULTS}

The results of the thermogravimetric tests were based on the analysis of the derivatograms. Fig. 8. presents a section of an example derivatogram for PC. The derivatogram shows the masschange curve - TG, the temperature-change curve $T$, the temperature-change differential curve DTA, and the mass-change differential curve - DTG. On the basis of the analysis of the functions, conclusions concerning the breakdown of chemical compounds contained in the samples that underwent the thermogravimetric analysis can be formulated. Analyzing the derivatogram for PC shown in fig. 6 ., it was concluded that its transition from the vitreous state to the high elasticity state occurs in a temperature of approx. $185^{\circ} \mathrm{C}$. Then, its slow gradual plasticizing can be observed. The onset of breakdown for this material occurs in a temperature of approx. $410{ }^{\circ} \mathrm{C}$; in $420{ }^{\circ} \mathrm{C}$ the breakdown is already evident; while in a temperature of $450{ }^{\circ} \mathrm{C}$, the breakdown process is at its most intense.

The derivatograms for the dye samples were analysed in a similar manner. It should be added that the tested dyes broke down in stages; however, due to the lack of data in the area of their chemical composition (manufacturer's secret), referenced were only the readings of temperatures corresponding to the first signs indicating the onset of breakdown of a complex chemical compound. Despite the fact that the curves were recorded in their entirety, as the chemical composition of the tested substance is not known, an in-depth, material analysis of the derivatograms is not possible. It is possible, though, to read the temperatures indicated by the DTA and DTG curves for the first signs indicating the first mass decrement and a change connected with the breakdown of material. Below, in table 1, the read characteristic temperatures for all the tested materials are summarized. It should be added that, as is commonly known, all plastics can be divided, on the basis of their structure and the physical and chemical properties, into amorphous and semicrystalline. The data provided by the contractor indicates that the received dyes were produced on the basis of polystyrene. This means that there are basically two kinds of materials being dealt with here, i.e. polycarbonate (PC), in the case of the base material, and polystyrene (PS), in the case of the dyes. In this context, it needs to be added that both materials belong to the group of amorphous materials, whose characteristic feature is that in the course of their cooling, from the plasticized to the solid state, their viscosity rapidly increases, making it difficult for the nuclei of crystallization to form and grow. This causes the lack of evident crystallization during the cooling of this type of materials, and the transition from the plasticized to the solid state is called the glass-transition point (temperature). Another name for materials of this type are 'supercooled liquids'. At the same time, during the heating of this type of materials, there is no transition through the melting point - only a transition into the state of high elasticity and the subsequent slow plasticizing.Regarding the data shown in table 1, it can be stated that a significant difference in the determined values of temperature between the base material and the dyes was noticed. Considering the temperature of the onset of breakdown in the case of $P C$ and dyes $\mathrm{G}$ and $\mathrm{B}$, the difference is approx. $100^{\circ} \mathrm{C}$, whereas in the case of $P C$ and dye 0 , approx. $150^{\circ} \mathrm{C}$. It can be stated that this proves the generally high temperature resistance of $P C$ and dyes $G$ and $B$. Only in the case of dye $O$ was a lower temperature of the onset of breakdown obtained. An important aspect, which may arouse suspicions as to the matrix of the medium of the dye, is the considerable difference between the temperature of the onset of breakdown for dye $\mathrm{O}$ and $\mathrm{G}$ and $\mathrm{B}$ dyes. This may mean that either other additives were used in the production process of this dye $(0)$, which modified its general thermal properties, or that another base was used. It should be noted here that according to the technological data, the tested dyes should be characterized by thermal stability up to approx. $300^{\circ} \mathrm{C}$.

Tab 1. The read values of characteristic temperature points

\begin{tabular}{|c|c|c|}
\hline $\begin{array}{c}\text { Sample } \\
\text { marking }\end{array}$ & $\begin{array}{c}\text { Glass-transition } \\
\text { temperature } \mathrm{T}_{\mathbf{z}} \\
{\left[{ }^{\circ} \mathrm{C}\right]}\end{array}$ & $\begin{array}{c}\text { Temperature of the } \\
\text { onset of breakdown } \\
{\left[{ }^{\circ} \mathrm{C}\right]}\end{array}$ \\
\hline PC & $175-185$ & $410-420$ \\
O & Reading failed & $255-260$ \\
G & $75-85$ & $305-310$ \\
B & $70-80$ & $315-320$ \\
\hline
\end{tabular}

Considering, however, the value of the processing temperature of this type of $P C\left(280-300{ }^{\circ} \mathrm{C}\right)$, then in relation to the temperature of breakdown of the dyes, the obtained values may indicate an insufficiently low margin of temperature. As is commonly known, during the plasticization process, the friction occurring between the molecules of the material, the slug and the cylinder walls leads to local high-temperature jumps, of even up to tens of degrees, which can lead to degradation of polymer structures. Assuming, thus, that the temperature on the individual cylinder and hot-channel zones (in the mold) reaches $300{ }^{\circ} \mathrm{C}$, then during plasticizing and injection it can locally rise by tens of degrees. The result of this fact is that all the assessed dyes exceed their thermal stability. This, on the other hand, may manifest itself by the dye being charred. This means that presence of black charred inclusions, which are the result of insufficient thermal stability of dyes, in the manufactured components during injection, is possible. Keeping in mind that dyes are usually mineral compounds of metal oxides with an exceptionally high temperature resistance (e.g. titanium white $\mathrm{TiO}_{2}$ : melting point 1850 ${ }^{\circ} \mathrm{C}$, boiling point $2500{ }^{\circ} \mathrm{C}$ ), it can be suspected that (in this particular case) the problem is connected with the medium. In other words, it can be supposed that the issue of visual and technological quality of PC-made components dyed with a PS-based additive is most probably related to the polystyrene medium - for the particular case. 


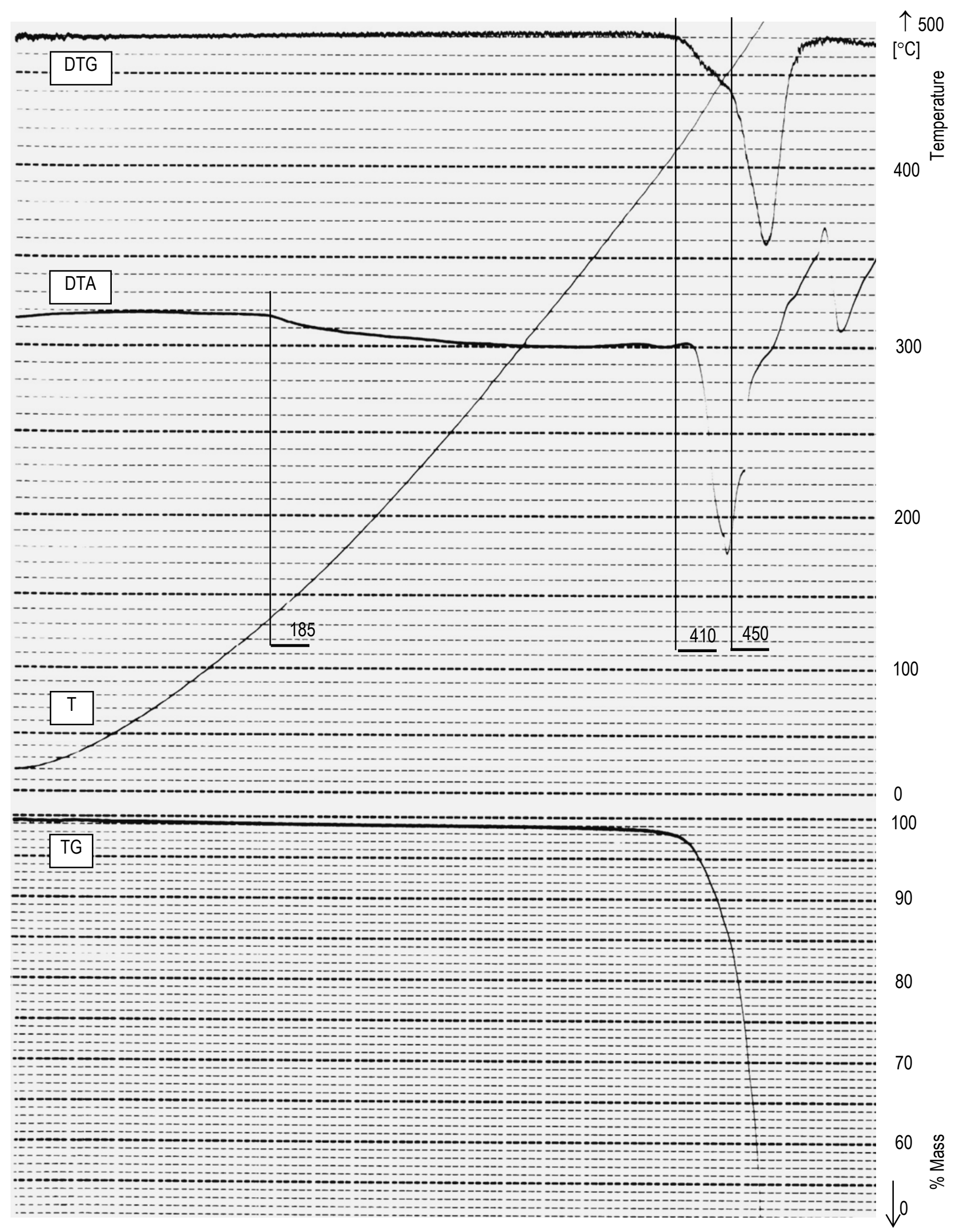

Fig. 8. An example derivatogram: TG- mass-change curve, T- temperature-rise curve, DTA- temperature-change differential curve, DTG- mass-change differential curve 
a)

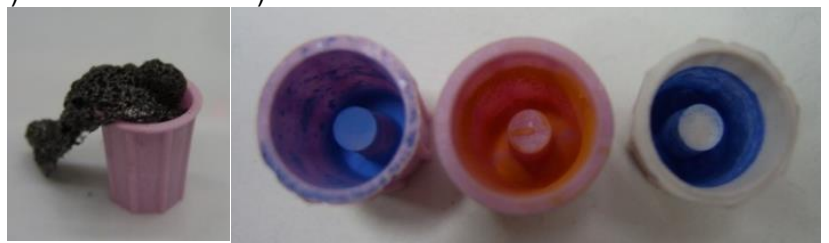

Fig. 9. View of the crucibles after derivatograph tests, remnants of: a) PC, b) B, c) O, d) G

The high temperature resistance of the pigments was confirmed in a general examination of the crucibles after the derivatograph tests. Fig. 9 shows photos of these crucibles.

As can be seen in the presented photos, PC was charred, but the maximum temperature in the derivatograph furnace $\left(500^{\circ} \mathrm{C}\right)$ was too high considering its temperature resistance, on the one hand; and too low to burn all the organics, on the other. This resulted in the appearance of a charred mass (Fig. 9a). In the case of the dyes, it turned out that the breakdown of these materials unfolded in such a way that no charred remnants of the process of their combustion were noticed. The matrix in which the color pigment was distributed had been burnt out earlier, due to its lower temperature resistance.

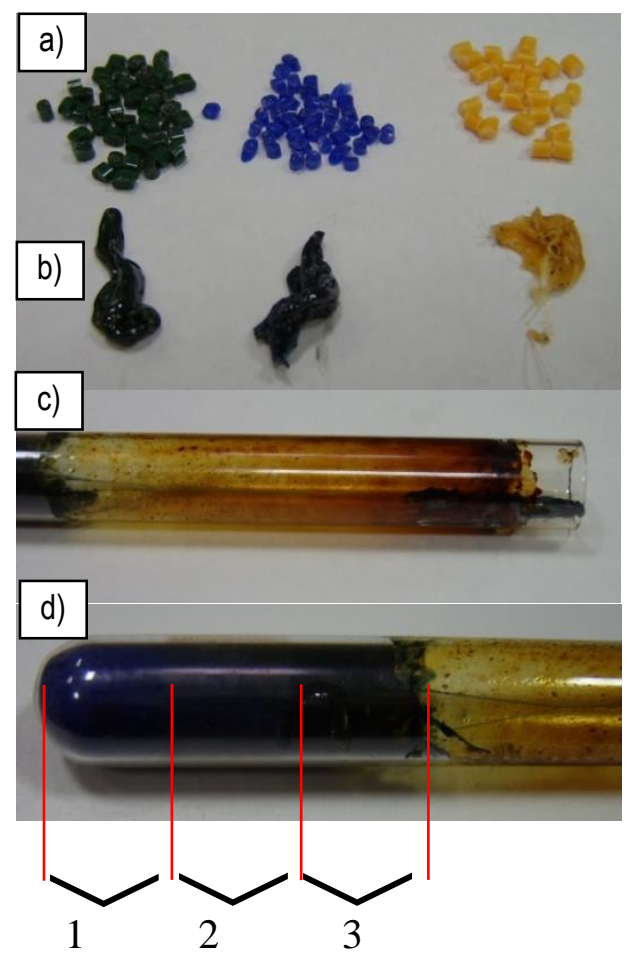

Fig. 10. Example photos of samples after the tests of thermal annealing $\left(300\left[{ }^{\circ} \mathrm{C}\right], \mathrm{t}=30\right.$ [min]): a) dye samples before the test, b) dye samples after annealing, $c$ ) view of the charred mass (B), d) view of dye $B$ after annealing tests; 1 - the area of pigment debris, 2- intermediate zone, 3 - charred medium zone

Referring back to the earlier statements concerning a faster burning of the medium used for the preparation of dyes, the following section of the research consists of tests of temperature resistance (thermal annealing) in lower temperatures (than those indicated by the temperatures of breakdown of the samples), but with the assumption that the material stays in the cylinder for a longer period of time. This state enables to simulate machine down time for the duration of, for example, short repair work. The tests in question were performed in a temperature of $280{ }^{\circ} \mathrm{C}$ for $60 \mathrm{~min}$, and in a temperature of $300^{\circ} \mathrm{C}$ for $30 \mathrm{~min}$. Below, in fig. 10 , are presented selected photos of dye samples after these tests.

a)

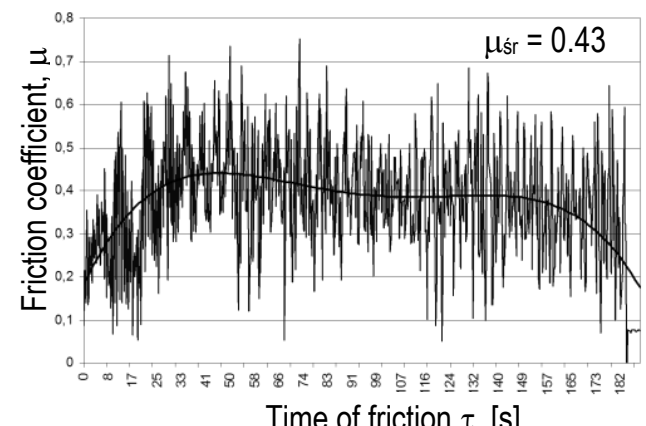

b)

Time of friction $\tau$, [s]
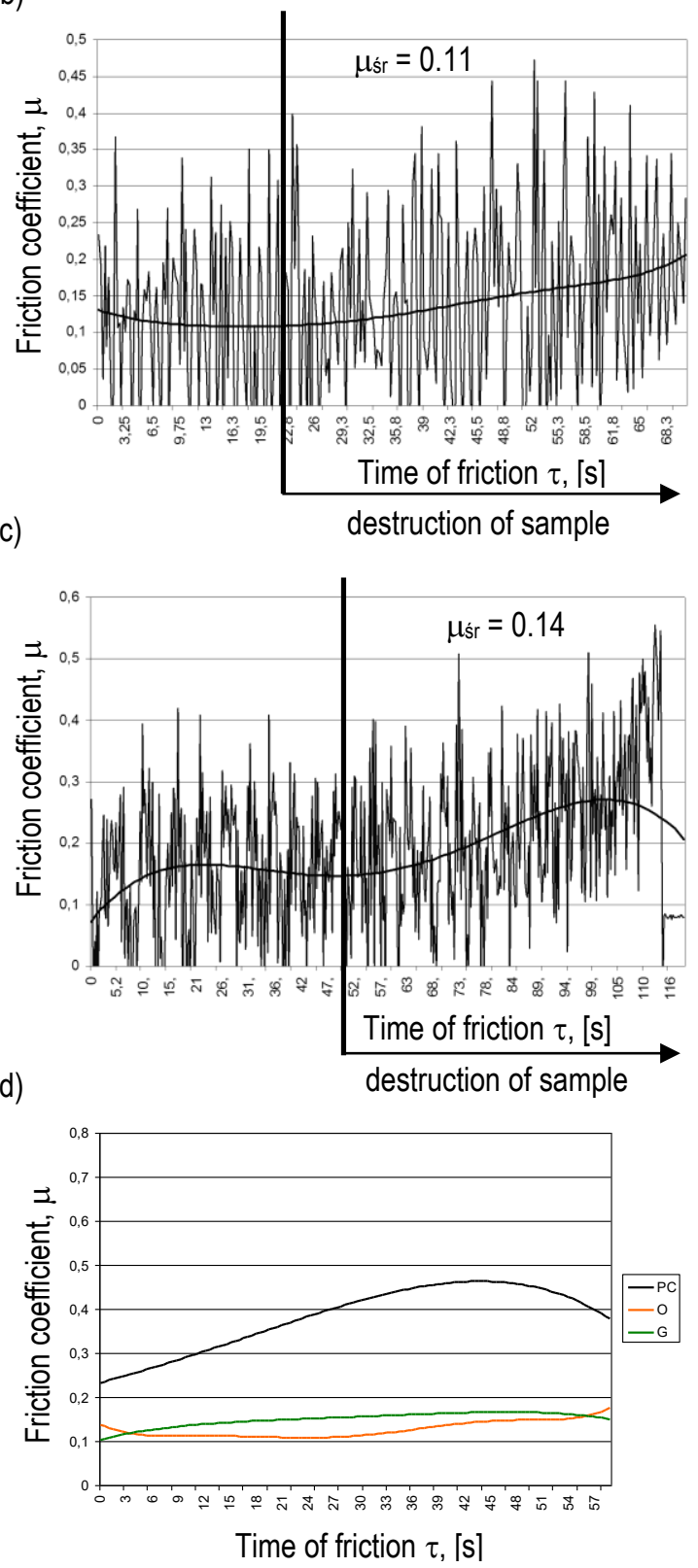

Fig. 11. Results of the tribological tests: a) for pure $P C, b)$ for dye 0 , c) for dye $G, d$ ) summary graph 
Figs. $10 a$ and $b$ show samples of the dyes before the test (Fig. 10a) and after the annealing tests (fig. 10b). The presented photos show that a 'good form' was retained only by dye 0 , notwithstanding that this is completely unconfirmed when considering the temperature of the onset of breakdown (255 - 260 $\left.{ }^{\circ} \mathrm{C}\right)$. In the case of dyes $B$ and $G$, it can be seen that the brightness of their color, especially in the case of dye $B$, was affected by products of degradation. The traces of combustion of the dye medium shown in Fig. $10 \mathrm{c}$ and $\mathrm{d}$ are even more visible. Fig. $10 \mathrm{C}$ shows the walls of the test tube in which dye $B$ was annealed. It can clearly be seen that the temperature of 300 ${ }^{\circ} \mathrm{C}$ and the time of annealing significantly influence the destruction of this additive. It can be added that in the case of dye $B$, after the annealing tests, phase borders between individual colored layers could be noticed in the test tube. The layer marked as 3 was a layer of charred material; the one marked as 1, a layer of intact dye; 2 - the layer isolated between the charred and the intact material.

There can obviously be objections concerning the conditions of the performed test; however, as mentioned earlier, friction between the molecules of the plasticized material; and between the walls of the cylinder, the slug and the material may locally lead to very high temperature jumps. With relation to this, Fig. 11 below shows the results of friction tests.

The results of these tests indicate lower values of the coefficient of friction for the dyes in comparison with PC. This means that in the conditions of the performed test, the dyes shear more easily, not causing a strong resistance to motion-related disturbance. In other words, it can be said that PC is a resistant material, not easily susceptible to shear stresses, which introduces additional disturbances (temperature rise) during the plasticization process. Keeping in mind that the value of the coefficient of friction for PC is approx. twice higher than for the dyes, a conclusion can surely be drawn that this fact is of considerable importance as far as friction heat, generated during the plasticization process, is concerned. This fact can be highly important concerning the visual quality of the manufactured components, due to the possibility of appearance of charred molecules of dye in their structure.

Describing the graphs shown in Fig. 11, it should be pointed out that in the case of PC, resistance to abrasive wear was high enough to enable to collect the test results throughout the entire duration of the performed experiment. In the case of the dyes, on the other hand (Fig. 11b and 11c), before the end of the experiment, a complete destruction of the sample collected for the tests was observed. This confirmed the low tribological resistance and easy shear of the tested dyes.

\section{CONCLUSIONS}

The tests carried out for the purpose of this paper and their analysis allowed to formulate the following general conclusions:

1. Lower temperatures of the onset of breakdown of the dyes in comparison with the base material were observed. It should be noted here that dye 0 was characterized by the lowest temperature that signified the onset of breakdown.

2. Keeping in mind the differences in the temperatures of breakdown for dyes $\mathrm{O}, \mathrm{G}$ and $\mathrm{B}$, it can be suspected that they were produced on different media, or the observed differences indicate dissimilarities in chemical composition between these dyes, which had a significant impact on the obtained results.
3. Keeping in mind the recommended processing temperatures of this type of $\mathrm{PC}\left(280-300^{\circ} \mathrm{C}\right)$ and the possibility of rapid local temperature jumps during the plasticizing and injection, it is considered that the obtained levels of temperatures of breakdown for $\mathrm{PC}\left(41{ }^{\circ} \mathrm{C}\right)$ and dyes $\mathrm{G}$ and $\mathrm{B}$ (305 and $31{ }^{\circ} \mathrm{C}$ ) are too low (small margin of temperature). The aforementioned local rise of temperature can cause a temperature rise by as much as tens of degrees in microareas of the plasticized alloy. This, in turn, may have an influence on local degradation of a, broadly understood, dye and determine the technological usability of components produced from this type of alloy. It should also be remembered that the temperature of breakdown for dye 0 was even lower $\left(255^{\circ} \mathrm{C}\right)$.

4. The performed tests of annealing in a constant temperature confirmed the misgivings (related to the dyes) concerning the kinetics of chemical reactions in the area of thermal resistance. It turned out that despite the fact that the obtained temperatures of breakdown were as high as over $300^{\circ} \mathrm{C}$, the analyzed additives broke down in lower temperatures in the case of a prolonged action of temperature. It should be noted here that the time of annealing of the dyes was $30 \mathrm{~min}$. This fact may be of importance in the case of machine down time and manifest itself in the form of degradation of dyes in conditions of normal injection molding processing. It is obvious that these kinds of problems can be overcome by repeatedly injecting 'fresh material' through the cylinder. There are areas of the injection space, however, where the flow of material may be limited, and this is where the charred remnants of dyes are likely to dwell.

5. The tribological tests of the analyzed materials proved that their friction characteristics can influence temperature rise during, for example, the plasticization process. It turned out that the results of friction tests indicate twice higher values of the coefficient of friction for PC in comparison with the dyes, which has a direct impact on the increase of alloy temperature and energy losses during the plasticization of PC.

6. Summarizing all the observations, it should be emphasized that when selecting dyes for the production of injection molded TS's, the compatibility of the dye and the polymer matrix should not be the sole factor taken under consideration. The thermal properties of the composition ingredients (range of processing temperatures and thermal breakdown) should also be scrutinized, as should, in special cases, other potentially relevant properties, e.g. the tribological properties of the ingredients. Production down time, which in many cases cannot be avoided, is also not without its relevance, as despite the sufficiently high processing temperatures, it can lead to degradation of material due to a prolonged action of high temperatures in the cylinder zones.

\section{REFERENCES}

1. Al-Salem SM, Lettieri P, Baeyens J. (2010), Recycling and recovery routes of plastic solid waste (PSW): A review, Waste Management, 29, 2625-2643.

2. Badia J.D., Francisco V., Sigbritt K., Ribes-Greus A. (2009), Thermal analysis as a quality tool for assessing the influence of thermo-mechanical degradation on recycled poly(ethylene terephthalate), Polymer Testing, 28(2), 169-175. 
3. Botos J., Murail N., Heidemeyer P., Kretschmer K., Ulmer B., Zentgraf T., Bastian M., Hochrein T. (2014), Color Measurement of Plastics - from Compounding via Pelletizing, up to Injection Molding and Extrusion, Proceedings of PPS-29: The 29th International Conference of The Polymer - Conference Papers, Book Series: AIP Conference Proceedings, V. 1593, 16-19.

4. Brachet P., Hoydal L.T., Hinrichsen E.L., Melum F. (2008), Modification of mechanical properties of recycled polypropylene from post-consumer containers, Waste Management, 28(12), 2456-2464.

5. Buccella M., Dorigato A., Crugnola F., Caldara M., Fambri L. (2015), Coloration Properties and Chemo-Rheological Characterization of a Dioxazine Pigment-Based Monodispersed Masterbatch, Journal Of Applied Polymer Science, 132(7), Article Number, 41452.

6. Chen W.-C., Nguyen M.-H., Chiu W.-H., Chen, T.-N., Tai, P.-H. (2016), Optimization of the plastic injection molding process using the Taguchi method, RSM, and hybrid GA-PSO, International Journal of Advanced Manufacturing Technology, 83(9-12), 1873-1886.

7. Chun DH. (1999), Cavity filling analyses of injection molding simulation: bubble and weld line formation, Journal of Materials Processing Technology, 90, 177-181.

8. Jałbrzykowski M. (2012), Influence of Regranulate Content on the Functional Properties of Parts Made from Thermoplastic Materials, Mach. Dyn. Res., 36(1), 82-89.

9. Jałbrzykowski M., Nachimowicz J. (2009), The problems of reliability and durability of tools for the injection of plastic materials, Acta Mechanica et Automatica, 3(1), 49-53.

10. Kayvon M.(2014), Sina Ebnesajjad and all: Handbook of Polymer Applications in Medicine and Medical Devices, William Andrew Inc., Elsevier, Oxford.
11. Laurence W. McKeen (2007), Effect of Temperature and other Factors on Plastics and Elastomers, William Andrew Inc., Elsevier, NY.

12. Matin I, Hadzistevic M, Hodolic J, Vukelic D, Lukic D (2012): A CAD/CAE-integrated injection mold design system for plastic products, International Journal Of Advanced Manufacturing Technology, 63(5-8), 595-607.

13. Navarro R., Torre L., Kenny J.M., Jiménez A. (2003), Thermal degradation of recycled polypropylene toughened with elastomers, Polymer Degradation and Stability, 82(2), 279-290.

14. Torres N., Robin J.J., Boutevin B. (2000), Study of thermal and mechanical properties of virgin and recycled poly(ethylene terephthalate) before and after injection molding, European Polymer Journal, 36(10), 2075-2080.

15. Wu C.-H., Chen W.-S. (2006), Injection molding and injection compression molding of three-beam grating of DVD pickup lens, Sensors and Actuators, Physical, 125 (2), 367-375.

16. Zawistowski H., Zięba Sz. (1999), Set the injection process, Plastech, Warsaw.

17. http://www.plastech.pl/pub/downloads/zytel-poradnik-przetworstwatworzywa-poliamidowe-dupont.pdf

18. http://www.wadim.com.pl/pl/a/Wady-wyprasek-Srodki-zaradcze-cz-1

19. https://www.elsevier.com/_data/assets/pdf_file/0011/91649/PlasticsUsed-in-Medical-Devices_link.pdf

20. www2.dupont.com/Poland_Country_Site/pl_PL/../10_zasad.html

This research was realized from statutory activity S/WM/1/14 and financed by MSHE. 